\title{
O-band InAs Quantum Dot Light Sources Monolithically Grown on Si
}

\author{
Shujie Pan, Mengya Liao, Zizhuo Liu, Ying Lu, Victoria Cao, Mingchu Tang, Jae-Seong Park, Jiang Wu, \\ Siming Chen*, Alwyn Seeds, and Huiyun Liu \\ Department of Electronic and Electrical Engineering, University College London, London WC1E $7 J E$ UK \\ E-mail address: siming.chen@ucl.ac.uk
}

\begin{abstract}
We discuss our recent progress made in the direct growth of $1.3 \mu \mathrm{m} \mathrm{InAs} / \mathrm{GaAs}$ quantum dot (QD) light-emitting sources on Si substrates for Si photonics. OCIS codes: (230.5590) Quantum-well, -wire and -dot devices; (250.5960) Semiconductor lasers; (250.5300) Photonic integrated circuits
\end{abstract}

\section{Introduction}

The availability of Si-based lasers is the key technology for the whole Si photonics industry. However, the indirect bandgap of $\mathrm{Si}$ is a severe limitation, and, despite recent advances, all-Si-based lasers will not, in the foreseeable future, outperform their III-V counterparts. Much effort has been directed toward hybrid integration of III-V lasers with Si photonics platforms. Although impressive results have been achieved, in the longer term, large-scale integration of photonics circuits will rely on the monolithic integration of laser sources on $\mathrm{Si}[1]$. The superiority of III-V quantum dot (QD) structures has attracted considerable attention over the last few decades for semiconductor lasers to achieve ultimately superior device performance [2], and has recently attracted increasing attention for monolithic III-V/Si integration due to their enhanced tolerance to defects [3].

In this paper, we first report our recent progress in the direct growth of $1.3 \mu \mathrm{m} \mathrm{InAs} / \mathrm{GaAs}$ QD lasers on $\mathrm{Si}$ substrates. We then move on to discuss the first study of post-growth fabrication and prototyping of various $\mathrm{Si}$ based InAs/GaAs QD light-emitting sources by utilizing the focused ion beam (FIB) technique.

\section{2. $1.3 \mu \mathrm{m}$ QD lasers grown on Si substrates}

To date, the fundamental road block limiting monolithic integration has been the large material dissimilarity between III-Vs and Si substrates. This discrepancy results in various types of defects, including antiphase boundaries (APBs), threading dislocations (TDs), and microcracks, which all generate non-radiative recombination centres and dramatically degrade the promise of III-V materials. To overcome this obstacle, the growth of a GaAs nucleation layer (NL) was first explored to reduce the density of TDs by effectively confining defects at the interface between the GaAs buffer and Si substrate. As a result, an electrically-pumped InAs/GaAs QD laser epitaxially grown on a Si substrate was demonstrated for the first time at $1.3 \mu \mathrm{m}$ under pulsed operation at room temperature (RT) with a threshold current density of $725 \mathrm{~A} / \mathrm{cm}^{2}$, an output power of $26 \mathrm{~mW}$ and lasing up to $42^{\circ} \mathrm{C}$ [4]. Soon after, a new type of AlAs NL was developed to further reduce the TDs within the GaAs epilayer on Si substrates. AlAs NL was found to be more effective in terms of confining defects within the AlAs/Si interface region and, consequently, fewer defects propagated into the GaAs buffer. The Sibased InAs/GaAs QD laser with an AlAs NL was demonstrated with a threshold current density of $650 \mathrm{~A} / \mathrm{cm}^{2}$ at RT and lasing up to $63^{\circ} \mathrm{C}$ [5]. To further minimize the impact of various types of defect, the combined strategies of an AlAs NL, InGaAs/GaAs DFLs [6], in situ thermal annealing [7] and using InAs QDs as the laser active regions have been developed to achieve high-quality III-V epilayers with a low density of threading dislocations on the order of $10^{5} \mathrm{~cm}^{-2}$. Based on this achievement, we have simultaneously achieved CW lasing up to $75^{\circ} \mathrm{C}$, with an ultralow $\mathrm{CW}$ threshold current density of $62.5 \mathrm{~A} / \mathrm{cm}^{2}$, a high output power exceeding 105 $\mathrm{mW}$ at RT and a long-extrapolated lifetime of over 100,158 h [8].

In our previous work mentioned above, to prevent the formation of APBs, off-cut Si (001) wafers have been used. While this approach can be successfully used for the annihilation of APBs, it compromises full compatibility with standard microelectronics fabrication, where on-axis $\mathrm{Si}(001)$ substrates are typically used. To overcome this barrier, the APB free epitaxial GaAs film with a small root-mean-square (RMS) surface roughness was first deposited on a 300-mm standard industry-compatible on-axis Si (001) substrate by metalorganic chemical vapor deposition (MOCVD). In doing so, an effective Si wafer preparation, as well as a twostep process, have been used [9]. The QD laser structure was then grown on this APB-free $\mathrm{GaAs} / \mathrm{Si}(001)$ virtual substrate by molecular beam epitaxy (MBE). Electrically-pumped CW $1.3 \mu \mathrm{m}$ InAs/GaAs QD lasers monolithically grown on on-axis $\mathrm{Si}(001)$ substrates without any intermediate buffer layers have been realized for the first time with a threshold current density of $425 \mathrm{~A} / \mathrm{cm}^{2}$ and single facet output power of $43 \mathrm{~mW}$. Under pulsed operation, lasing operation up to $102^{\circ} \mathrm{C}$ has been realized, with a threshold current density of $250 \mathrm{~A} / \mathrm{cm}^{2}$ and single facet output power exceeding $130 \mathrm{~mW}$ at RT [10].

\section{Post-fabrication of III-V Light Sources on Si by FIB Technique}


While optical lithography using photoresist is the dominant patterning technique, as it allows the patterning of an entire $300 \mathrm{~mm}$ (and larger) wafer in a short time with high yield, due to the need for expensive masks and repeated processing, development of new devices is costly and time-consuming. It is therefore attractive to use prototyping technologies that enable rapid and flexible fabrication of nanophotonic components, ranging from micro- to nanometer scales. Among these approaches, FIB is an interesting alternative as it allows photoresistfree and direct writing, which enables the post-fabrication of devices with a more complex topography such as ridge waveguides and laser facets. Also, FIB technology has been widely used for photonics applications, including fabrication of grating couplers and various waveguides.

To this end, the first study of post-fabrication and prototyping of various Si-based light emitting sources has been demonstrated by utilizing the FIB technique, with the intention of expediting the progress toward largescale and low-cost photonic integrated circuits monolithically integrated on a Si platform. Lasing characteristics for silicon-based lasers with FIB-created facets and as-cleaved facets were compared, and the initial results reveal that there was no obvious deterioration between these two facet realization approaches. Various Si-based light emitting sources, such as lasers, superluminescent diodes (SLDs) [11] and LEDs, were then realized by effectively reduced facet reflectivity using focused Ga+ion beam milling of the front facet of the edge emitting Si-based InAs/GaAs QD laser [12].

\section{Conclusion}

We have discussed our recent progress in III-V lasers monolithically grown on Si substrates with offcut and standard industry-compatible on-axis $\mathrm{Si}(001)$ substrates. We have also reported the first studies of postfabrication of various Si-based III-V QD light sources by means of FIB for use in Si photonics.

\section{Reference}

[1] E. Tournié, L. Cerutti, J.-B. Rodriguez, H. Liu, J. Wu, and S. Chen, "Metamorphic III-V semiconductor lasers grown on silicon,” MRS Bulletin 41, 218-223 (2016).

[2] M. Sugawara and M. Usami, "Handling the heat," Nature Photonics 3, 30-31 (2009).

[3] A. Y. Liu, S. Srinivasan, J. Norman, A. C. Gossard, and J. E. Bowers, "Quantum dot lasers for Siphotonics [Invited]," Photonics Research. 3, B1-B9 (2015).

[4] T. Wang, H. Liu, A. Lee, F. Pozzi, and A. Seeds, "1.3- $\mu \mathrm{m}$ InAs/GaAs quantum-dot lasers monolithically grown on Si substrates," Optics Express 19, 11381-11386 (2011).

[5] A. D. Lee, Qi Jiang, Mingchu Tang, Yunyan Zhang, A. J. Seeds, and Huiyun Liu, "InAs/GaAs Quantum-Dot Lasers Monolithically Grown on Si, Ge, and Ge-on-Si Substrates,” IEEE J. Sel. Top. Quantum Electron 19, 1901107-1901107 (2013).

[6] M. Tang et al., "Optimizations of Defect Filter Layers for 1.3- $\mu \mathrm{m}$ InAs/GaAs Quantum-Dot Lasers Monolithically Grown on Si Substrates," IEEE J. Sel. Top. Quantum Electron 22, 50-56 (2016).

[7] J. R. Orchard et al., "In situ annealing enhancement of the optical properties and laser device performance of InAs quantum dots grown on Si substrates," Optics Express 24, 6196-6202 (2016).

[8] S. Chen et al., "Electrically pumped continuous-wave III-V quantum dot lasers on silicon,” Nature Photonics 10, 307-311 (2016).

[9] R. Alcotte et al., "Epitaxial growth of antiphase boundary free GaAs layer on $300 \mathrm{~mm} \mathrm{Si(001)} \mathrm{substrate} \mathrm{by} \mathrm{metalorganic} \mathrm{chemical}$ vapour deposition with high mobility," APL Materials 4, 46101 (2016).

[10] S. Chen et al., "Electrically pumped continuous-wave $13 \mu \mathrm{m}$ InAs/GaAs quantum dot lasers monolithically grown on on-axis Si (001) substrates," Optics Express 25, $4632-4639$ (2017).

[11] S. Chen et al., "InAs/GaAs Quantum-Dot Superluminescent Light-Emitting Diode Monolithically Grown on a Si Substrate," ACS Photonics 1, 638-642 (2014).

[12] M. Liao et al., "Monolithically Integrated Electrically Pumped Continuous-Wave III-V Quantum Dot Light Sources on Silicon," IEEE J. Sel. Top. Quantum Electron. 23, 1-10 (2017). 